\title{
DO OLDER WOMEN WITH A FALL HISTORY CROSS OBSTACLES DIFFERENTLY THAN OLDER WOMEN WITH NO FALL HISTORY?
}

original paper

( ) University School of Physical Education in Wroclaw

DOI: https://doi.org/10.5114/hm.2020.91345

\section{ELIANE C. GUADAGNIN ${ }^{1}$, EMMANUEL S. DA ROCHA ${ }^{2}$, MAARTEN F. BOBBERT ${ }^{3}$, JACQUES DUYSENS ${ }^{4}$, FELIPE P. CARPES ${ }^{1}$}

${ }^{1}$ Applied Neuromechanics Research Group, Laboratory of Neuromechanics, Federal University of Pampa, Uruguaiana, Brazil

${ }^{2}$ Biomechanics and Kinesiology Research Group, Exercise Research Laboratory, Federal University of Rio Grande do Sul, Porto Alegre, Brazil

${ }^{3}$ Department of Human Movement Sciences, Faculty of Behavioural and Movement Sciences,

VU University Amsterdam, Amsterdam, The Netherlands

${ }^{4}$ Department of Movement Sciences, Faculty of Movement and Rehabilitation Sciences, KU Leuven, Heverlee, Belgium

\begin{abstract}
Purpose. Many falls in older people occur after tripping or slipping, mainly due to unsuccessful vertical clearances or horizontal distances. A first fall may be explained by several factors related to aging and can be a trigger to subsequent falls. It is unclear if a history of fall changes the kinematics of obstacle crossing, increasing the risk of trips. Here, we determined whether older women reporting a fall history showed different spatial-temporal kinematic parameters during obstacle crossing than non-fallers. In addition, we investigated the presence of asymmetries between the preferred and non-preferred lower limb during obstacle crossing in fallers and non-fallers.

Methods. This cross-sectional study recruited older women with a history of fall $(n=10)$ and without falls $(n=10)$. They had their kinematic parameters evaluated when walking at self-selected speed along an 8-m walkway, crossing an obstacle positioned in the middle of the walkway, with both preferred and non-preferred limb as the lead limb. The groups were compared, and effects of lower limb preference were also determined in both groups.

Results. No main effects of group were observed regarding the kinematic variables. An effect of leg preference was found for post-obstacle horizontal distance, which was greater for the preferred limb in both groups.

Conclusions. In conclusion, the kinematics of gait with obstacle crossing does not differentiate between older women with or without a history of recent fall.
\end{abstract}

Key words: aging, gait, tripping, kinematics

\section{Introduction}

Many professionals are interested in tools that permit to identify differences between fallers and nonfallers when it comes to designing fall prevention programs for older people and minimizing hazards in daily life. Previous studies investigated differences in the kinematics of unobstructed gait between older fallers and non-fallers [1-4]. Some research has shown that people who have already fallen present a different gait pattern than non-fallers [1-3, 5, 6], but it seems that these spatial-temporal characteristics are not enough to differentiate these groups during level gait and do not predict falls [4], despite the relationship with functional performance [7].

Unintentional falls are among the leading causes of accidents and injuries involving older people and are more likely to occur after tripping or slipping [8], resultant of unsuccessful lower toe clearance [9] or putting the feet too close to the obstacle before or after crossing [10]. Most previous studies defined a faller as someone who had experienced at least one fall during the preceding year [4], and in terms of sex, more women (28.4\%) than men (8.4\%) reported falling [11].

Correspondence address: Felipe P. Carpes, Federal University of Pampa, Laboratory of Neuromechanics, BR 472 km 592 -

Po box 118 - ZIP 97500-970, Uruguaiana, RS, Brazil, e-mail: carpes@unipampa.edu.br

Received: March 29, 2019

Accepted for publication: November 2, 2019

Citation: Guadagnin EC, da Rocha ES, Bobbert MF, Duysens J, Carpes FP. Do older women with a fall history cross obstacles differently than older women with no fall history? Hum Mov. 2020;21(3):47-53; doi: https://doi.org/10.5114/hm.2020.91345. 
Those previous studies analysed temporal (cadence, stride time, duration of single and double support, walking speed) and spatial (stride length, step length, step width, and gait variability) parameters of gait. However, there is little information regarding the comparison of fallers and non-fallers during gait with obstacle crossing, a more challenging task, commonly experienced during daily life [4]. For this reason, a kinematic study of obstacle crossing is a useful paradigm to assess fall risk in older adults. In addition, lower limb asymmetries during obstacle crossing are an important aspect to be investigated in older individuals, once that those classified as with high risk of falls were shown to be asymmetric during obstacle crossing [12]. However, this analysis was done only during gait initiation [12].

When considering gait with obstacles, an increased knee flexion during walking and small steps prior to obstacle clearance (pre-obstacle horizontal distance) were observed in fallers compared with non-fallers [5]. Additionally, when submitted to a protocol of perturbed gait, older people who had fallen after the perturbation had lower knee strength than those who had not previously fallen [13]. Fallers have also a worse anticipatory postural adjustment on gait initiation for obstacle crossing [14]. However, there are still few results in the literature on obstacle crossing during overground gait comparing independent older adults without a history of fall with those who had fallen in the previous months.

The aim of the present study was to determine whether older women with a fall history would show different gait kinematics during obstacle crossing than older women without a history of falls. In addition, we investigated if there were asymmetries between the preferred and non-preferred lower limbs during obstacle crossing in fallers and non-fallers. Our hypothesis was that the older individuals with a recent history of fall would present worse kinematic gait parameters (e.g. smaller clearances) compared with nonfallers and that only the fallers would present asymmetries between lower limbs.

\section{Material and methods}

\section{Experimental design}

This is a cross-sectional study in which older adults with and without a history of fall walked at self-selected speed along an 8-m walkway, in the middle of which they had to negotiate an obstacle. Kinematic data were collected to determine gait speed as well as bilateral step length, pre-obstacle and post-obstacle horizontal distances, and heel and toe clearances at specific moments during obstacle crossing. The groups were compared, and effects of lower limb preference were determined.

\section{Participants}

The volunteers participating in this study were ablebodied older women. They were able to walk independently, had good comprehension of instructions during the experiment, with a score higher than 22 points in the Mini-Mental State Examination (MMSE) [15].

The subjects were asked if they had fallen in the previous 12 months (i.e. Have you fallen in the past 12 months?); the context of the falls was also investigated. Information on fall history was collected during a comprehensive interview. The participants who had fallen at least once in the preceding 12 months during standing or walking (falls that occurred in the static sitting or lying position were not considered) were assigned to a group of 'fallers' ( $n=10$; age: $66 \pm 5$ years; body mass: $63.5 \pm 13.4 \mathrm{~kg}$; height: $1.53 \pm 0.05 \mathrm{~m}$; MMSE: $26.3 \pm 2.2$ points; number of falls in the past year: $1.6 \pm 1.3$ ), and those without falls formed a group of 'non-fallers' $(n=10$; age: $66 \pm$ 4 years; body mass: $72.3 \pm 14.2 \mathrm{~kg}$; height: $1.55 \pm$ $0.07 \mathrm{~m}$; MMSE: $27.1 \pm 2.1$ points). The groups did not differ in age (independent $t$-test: $p=0.846$ ), body mass $(p=0.172)$, height ( $p=0.421)$, or MMSE score $(p=0.416)$. The revised Waterloo footedness questionnaire [16] indicated the right lower limb as the preferred limb in all participants.

\section{Gait assessment}

Spherical retroreflective markers were attached to the participants' body surface in accordance with the Plug-in Gait full body model (Vicon Motion Systems, Oxford, UK). Additional retroreflective markers were attached on both halluces and on the 4 edges of the obstacle. Kinematics was sampled at $200 \mathrm{~Hz}$ by using 6 infrared cameras (Vicon Motion Systems, Oxford, UK). Data were low-pass filtered with a $4^{\text {th }}$ order Butterworth filter with cut-off frequency of $8 \mathrm{~Hz}$.

The participants were requested to walk barefoot at self-selected speed along an 8-m walkway. In the middle of the walkway, they crossed a polystyrene obstacle (length $\times$ width: $80 \times 20 \mathrm{~cm}$; height defined as $20 \%$ of the participant's lower limb length, determined by the vertical distance from the greater trochanter to the ground during upright standing). 
After a familiarization trial, crossings with either preferred or non-preferred lower limb leading were elicited by having participants initiate their gait from a standing position with either the left or the right lower limb and varying the distance from the obstacle. Ten trials were recorded for each lower limb crossing the obstacle. Lower limb asymmetries were considered when there were kinematic differences between the preferred and non-preferred limb during obstacle crossing.

The kinematic measures related to fall risk investigated in the present study were: average gait speed along the 8-m walkway, toe clearance in leading limb (LL) and trailing limb (TL) (vertical distance between the hallux and the obstacle, measured when the hallux was at the lowest horizontal distance from the obstacle marker), step length in LL and TL (measured during the crossing stride only), LL heel clearance (vertical distance between the heel and the obstacle, determined at the instant that the heel was at the lowest horizontal distance from the obstacle marker), pre-obstacle horizontal distance (horizontal distance between hallux and obstacle before crossing), and postobstacle horizontal distance (horizontal distance between heel and obstacle after crossing), as determined in a previous study [17].

\section{Statistical analyses}

Data were tested for normality by using the ShapiroWilk test. An analysis of variance (two-way ANOVA) with Bonferroni correction was performed to verify effects and interactions for group (with and without fall) and lower limb (preferred and non-preferred). All tests were carried out with the SPSS 20.0 software. The significance level was set at 0.05 .

\section{Ethical approval}

The research related to human use has complied with all the relevant national regulations and institutional policies, has followed the tenets of the Declaration of Helsinki, and has been approved by the Federal University ofPampa ethics committee(IRB\#0172011).

\section{Informed consent}

Informed consent has been obtained from all individuals included in this study.

\section{Results}

Obstacle contacts were observed in $1 \%$ of the trials and none of them resulted in a fall. The frequency of obstacle contacts within the $1 \%$ of the trials was similar between the groups. These trials were excluded from data analysis. Group effects or interactions were not found (Table 1 and Figure 1). Gait speed did not differ between the groups. A main effect of lower limb preference was noted for post-obstacle horizontal distance $\left(F_{(1)}=5.691 ; p=0.041\right)$, being greater for the preferred limb. The similarity in gait kinematics between the groups can also be observed in Figure 2, where we illustrate the average patterns in the different phases of obstacle crossing for the participants from each group.

\section{Discussion}

Tripping over objects is considered an important cause of falls in older adults [18]. Here we found that older women with a history of falls (at least one fall in the previous year) presented similar gait kinematics during obstacle crossing as those without a history of falls. No differences in toe and heel clearances were

Table 1. Statistical parameters pertaining to obstacle crossing in the older women with and without a history of fall

\begin{tabular}{lcccccc}
\hline \multirow{2}{*}{ Variables } & \multicolumn{2}{c}{ Group } & & Lower limb & \multicolumn{3}{c}{ Group $\times$ lower limb } \\
\cline { 2 - 6 } & $F$ & $p$ & $F$ & $p$ & $F$ & 0.032 \\
\hline LL toe clearance & 0.004 & 0.952 & 0.005 & 0.946 & 0.862 \\
TL toe clearance & 1.217 & 0.299 & 0.002 & 0.962 & 0.059 & 0.813 \\
LL heel clearance & 1.380 & 0.270 & 0.325 & 0.583 & 1.073 & 0.327 \\
Pre-obstacle horizontal distance & 0.259 & 0.623 & 0.001 & 0.982 & 0.007 & 0.937 \\
Post-obstacle horizontal distance & 4.750 & 0.057 & 5.691 & $0.041^{*}$ & 0.906 & 0.366 \\
Gait speed & 0.501 & 0.497 & 0.302 & 0.596 & 0.017 & 0.899 \\
LL step length & 0.046 & 0.835 & 2.419 & 0.154 & 2.455 & 0.152 \\
TL step length & 2.901 & 0.123 & 3.438 & 0.097 & 1.143 & 0.313 \\
\hline
\end{tabular}

$F$ and $p$ values observed for main effects of group and lower limb and for interactions between them; LL - leading limb,

$\mathrm{TL}$ - trailing limb

* mean statistical significance $(p<0.05)$ 


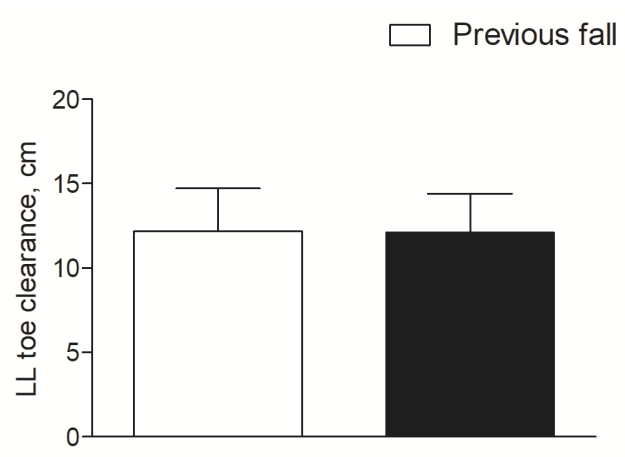

No previous fall
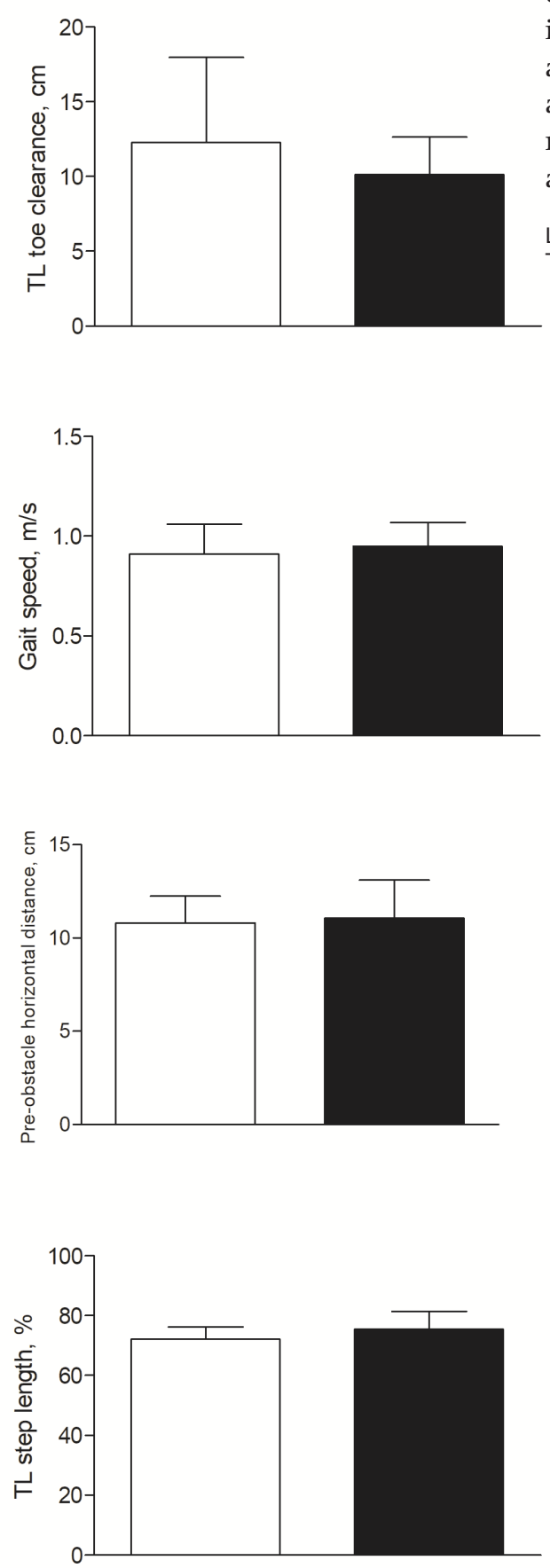

observed between the groups. A previous review reported that stance time variability, gait speed, stride length, and step length were the spatial-temporal parameters most different between elderly fallers and non-fallers. It may suggest that using only spatial-temporal analysis of level walking might not be sufficient and reliable to predict falls in older people[4]. In a previous investigation involving gait initiation and obstacle crossing, and considering the presence of bilateral asymmetries, older fallers presented greater asym-
Figure 1. Kinematic variables data determined during obstacle crossing in the older women with and without a history of fall. Data from the preferred and non-preferred lower limbs were merged and are expressed as means and standard deviations

$\mathrm{LL}$ - leading limb

$\mathrm{TL}$ - trailing limb
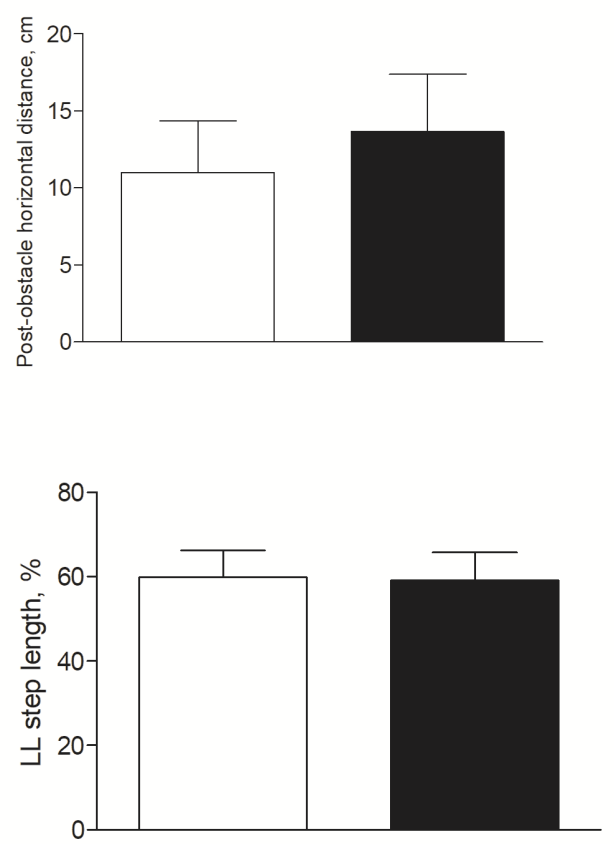

metries for foot clearance than non-fallers [12]. However, in the present study, when the participants needed to cross the obstacle when already walking, lower limb asymmetry was not found for toe clearances. There was a difference, similar for both groups, only between lower limbs for post-obstacle horizontal distance.

The groups did not differ regarding the self-selected gait speed, which may be surprising. However, despite the fact that gait speed seems to be one of the more important clinical markers of health in older adults [19], 

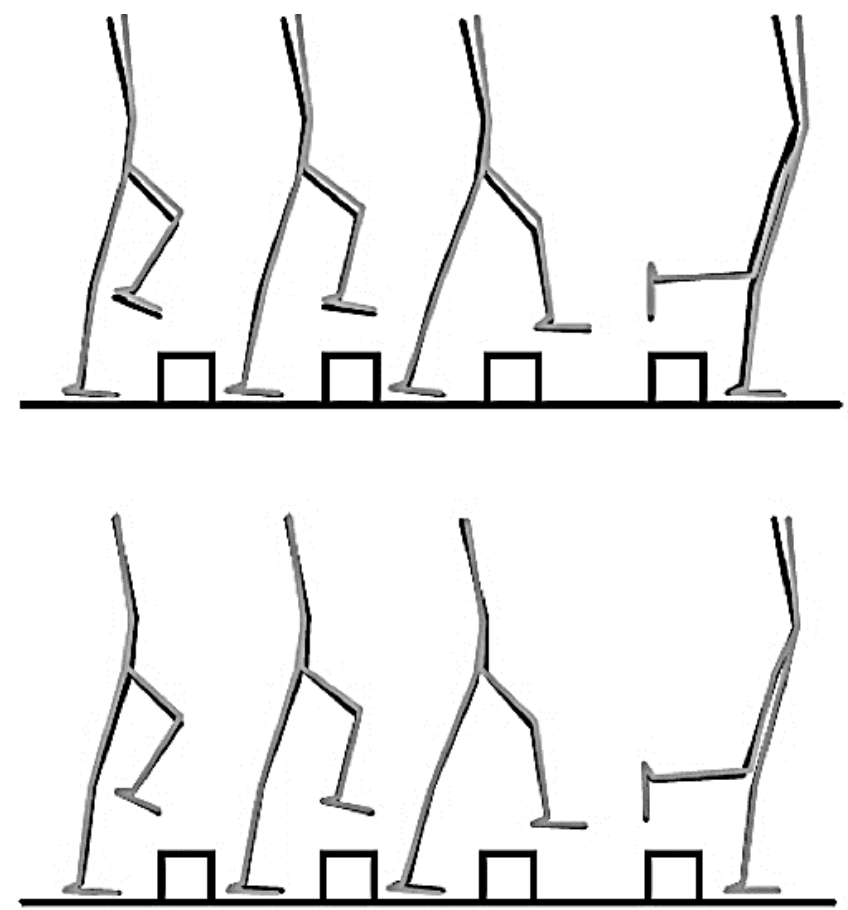

Figure 2. Stick representative diagrams for kinematic parameters during obstacle crossing in the groups of fallers and non-fallers. The average results for each group are presented. In the top diagram, the data are

representative for obstacle approach with the nonpreferred limb, and in the bottom one, for the approach with the preferred limb. Black lines denote non-fallers and silver lines indicate fallers

previous studies showed conflicting results: some reported no differences when comparing fallers and non-fallers [1, 20, 21], while others presented slower gait among fallers [22, 23]. Often, these discrepancies may be related to differences in populations, for example inclusion of cognitively impaired older people [23]. The older individuals in our study were independent, and this may help to explain the lack of difference in gait speed. Furthermore, we considered an obstacle height related to the individual lower limb length, which may have created a challenge condition more uniform for the different participants.

An increased post-obstacle horizontal distance could be related to a lower risk of heel contact with the obstacle, and therefore was considered an important variable to measure in terms of success rates in obstacle crossing. However, both groups showed a similar behaviour for this parameter, with an absolute difference lower than $2 \mathrm{~cm}$. Furthermore, it is interesting to note that while toe clearance is suggested the main variable related to tripping, we did not observe any difference in this parameter when comparing the groups.
It is always a point of discussion whether a smaller [17] or a larger [24] toe clearance elicits risk of obstacle contacts, and it has been argued that an obstacle contact leading to a trip commonly results from insufficient foot clearance. The insufficient clearance may come from the fact that perhaps people who know what they are doing have small clearance and higher confidence, and people who are insecure have large clearance but low confidence, causing them to hit the obstacle. The use of smaller step lengths can lead to smaller post-obstacle horizontal distances, resulting in the foot landing at a lower distance behind the obstacle. Both strategies are used more frequently with aging [25] and, theoretically, a larger post-obstacle horizontal distance may appear as an advantage because of reduced risk of obstacle contact. However, as pointed out by Muir et al. [25], there is a price to pay. A longer step may be destabilizing because of the long swing during single support. The perceived imbalance could make older individuals put their lead foot sooner to the ground [25]. Another possibility is that it is not a conscious strategy but simply a result of biomechanical constraints (e.g. reduced hip range of motion) [25-28].

Our study has limitations. We screened a group of 42 elderly as part of a research project and included here only 10 individuals with previous falls. They were paired to 10 other elderly randomly picked from the main group of subjects. It is important to note that the retrospective method to classify an older individual as a faller or a non-faller and to determine the number of falls can be imprecise, and this is a limitation of our study. We considered only the sagittal kinematics parameters because a large range of motion for walking is observed at this plane, and finally, we did consider the angular parameters because our focus was dedicated to the risk of obstacle contact that resulted in tripping.

\section{Conclusions}

Fallers and non-fallers did not differ in all obstacle crossing kinematics considered. Owing to the lack of difference in kinematic parameters, we suggest that sagittal plane kinematics of gait with obstacle crossing could not differentiate between older women with or without a history of recent fall.

\section{Acknowledgements}

This study was partially financed by the Coordination for the Improvement of Higher Education Personnel (CAPES, Coordenação de Aperfeiçoamento de Pessoal de Nível Superior), Brazil, Finance Code 001 - for ECG 
and ESR, by the CNPq - for FPC, and by Science Without Borders CNPq - for MB and JD. Authors acknowledge the support received from FAPERGS during the development of this research.

\section{Disclosure statement}

No author has any financial interest or received any financial benefit from this research.

\section{Conflict of interest}

The authors state no conflict of interest.

\section{References}

1. Cebolla EC, Rodacki AL, Bento PC. Balance, gait, functionality and strength: comparison between elderly fallers and non-fallers. Braz J Phys Ther. 2015;19(2):146151; doi: 10.1590/bjpt-rbf.2014.0085.

2. Barrett RS, Mills PM, Begg RK. A systematic review of the effect of ageing and falls history on minimum foot clearance characteristics during level walking. Gait Posture. 2010;32(4):429-435; doi: 10.1016/j.gaitpost. 2010.07.010.

3. Studenski S, Perera S, Patel K, Rosano C, Faulkner K, Inzitari M, et al. Gait speed and survival in older adults. JAMA. 2011;305(1):50-58; doi: 10.1001/jama.2010.1923.

4. Mortaza N, Abu Osman NA, Mehdikhani N. Are the spatio-temporal parameters of gait capable of distinguishing a faller from a non-faller elderly? Eur J Phys Rehabil Med. 2014;50(6):677-691.

5. Newstead AH, Walden JG, Gitter AJ. Gait variables differentiating fallers from nonfallers. J Geriatr Phys Ther. 2007;30(3):93-101; doi: 10.1519/00139143200712000-00003.

6. Abellan van Kan G, Rolland Y, Andrieu S, Bauer J, Beauchet O, Bonnefoy M, et al. Gait speed at usual pace as a predictor of adverse outcomes in community-dwelling older people an International Academy on Nutrition and Aging (IANA) Task Force. J Nutr Health Aging. 2009;13(10):881-889; doi: 10.1007/s12603009-0246-z.

7. Sadowska D, Gumny M, Osiński W. Are the timed up and go test and functional reach test useful predictors of temporal and spatial gait parameters in elderly people? Hum Mov. 2016;17(3):148-153; doi: 10.1515/humo2016-0025.

8. Berg WP, Alessio HM, Mills EM, Tong C. Circumstances and consequences of falls in independent community-dwelling older adults. Age Ageing. 1997;26(4):261268; doi: 10.1093/ageing/26.4.261.

9. Soma M, Masuda T, Shimamura R, Abiko T, Uematu H, Kawama K. Influence of a dual-task on toe clearance of the young and elderly while stepping over an obstacle. JPhys Ther Sci. 2010;22(1):75-79; doi: 10.1589/jpts.22.75.

10. Weerdesteyn V, Nienhuis B, Duysens J. Advancing age progressively affects obstacle avoidance skills in the elderly. Hum Mov Sci. 2005;24(5-6):865-880; doi: 10.1016/j.humov.2005.10.013.

11. Gazibara T, Kurtagic I, Kisic-Tepavcevic D, Nurkovic S, Kovacevic N, Gazibara T, et al. Falls, risk factors and fear of falling among persons older than 65 years of age. Psychogeriatrics. 2017;17(4):215-223; doi: 10.1111/ psyg.12217.

12. Di Fabio RP, Kurszewski WM, Jorgenson EE, Kunz RC. Footlift asymmetry during obstacle avoidance in highrisk elderly. J Am Geriatr Soc. 2004;52(12):20882093; doi: 10.1111/j.1532-5415.2004.52569.x.

13. Ding L, Yang F. Muscle weakness is related to slip-initiated falls among community-dwelling older adults. J Biomech. 2016;49(2):238-243; doi: 10.1016/j.jbiomech.2015.12.009.

14. Uemura K, Yamada M, Nagai K, Ichihashi N. Older adults at high risk of falling need more time for anticipatory postural adjustment in the precrossing phase of obstacle negotiation. J Gerontol A Biol Sci Med Sci. 2011;66(8):904-909; doi: 10.1093/gerona/glr081.

15. Folstein MF, Folstein SE, McHugh PR. "Mini-mental state”. A practical method for grading the cognitive state of patients for the clinician. J Psychiatr Res. 1975; 12(3):189-198; doi: 10.1016/0022-3956(75)90026-6.

16. Elias LJ, Bryden MP, Bulman-Fleming MB. Footedness is a better predictor than is handedness of emotional lateralization. Neuropsychologia. 1998;36(1): 37-43; doi: 10.1016/s0028-3932(97)00107-3.

17. Guadagnin EC, da Rocha ES, Mota CB, Carpes FP. Effects of regular exercise and dual tasking on spatial and temporal parameters of obstacle negotiation in elderly women. Gait Posture. 2015;42(3):251-256; doi: 10.1016/j.gaitpost.2015.05.012.

18. Tinetti ME, Speechley M, Ginter SF. Risk factors for falls among elderly persons living in the community. N Engl J Med. 1988;319(26):1701-1707; doi: 10.1056/ NEJM198812293192604.

19. Fritz S, Lusardi M. White paper: "walking speed: the sixth vital sign”. J Geriatr Phys Ther. 2009;32(2):46-49; doi: 10.1519/00139143-200932020-00002.

20. Freire Júnior RC, Porto JM, Marques NR, Magnani PE, Abreu DC. The effects of a simultaneous cognitive or motor task on the kinematics of walking in older fallers and non-fallers. Hum Mov Sci. 2017;51:146-152; doi: 10.1016/j.humov.2016.12.004.

21. Wright RL, Peters DM, Robinson PD, Watt TN, Hollands MA. Older adults who have previously fallen due to a trip walk differently than those who have fallen due to a slip. Gait Posture. 2015;41(1):164-169; doi: 10.1016/j.gaitpost.2014.09.025.

22. Kemoun G, Thoumie P, Boisson D, Guieu JD. Ankle dorsiflexion delay can predict falls in the elderly. J Rehabil Med. 2002;34(6):278-283; doi: 10.1080/165019 702760390374.

23. Taylor ME, Delbaere K, Mikolaizak AS, Lord SR, Close JC. Gait parameter risk factors for falls under simple and dual task conditions in cognitively impaired 
older people. Gait Posture. 2013;37(1):126-130; doi: 10.1016/j.gaitpost.2012.06.024.

24. Pan H-F, Hsu H-C, Chang W-N, Renn J-H, Wu H-W. Strategies for obstacle crossing in older adults with high and low risk of falling. J Phys Ther Sci. 2016;28(5): 1614-1620; doi: 10.1589/jpts.28.1614.

25. Muir BC, Haddad JM, Heijnen MJ, Rietdyk S. Proactive gait strategies to mitigate risk of obstacle contact are more prevalent with advancing age. Gait Posture. 2015;41(1):233-239; doi: 10.1016/j.gaitpost.2014.10.005.

26. Christensen JC, Wilson CR, Merryweather AS, Foreman KB. Kinematics of the pelvis, torso, and lower limb during obstacle negotiation while under temporal constraints. Anat Rec. 2017;300(4):732-738; doi: 10.1002/ar.23554.

27. Chou LS, Draganich LF. Placing the trailing foot closer to an obstacle reduces flexion of the hip, knee, and ankle to increase the risk of tripping. J Biomech. 1998;31(8): 685-691; doi: 10.1016/s0021-9290(98)00081-5.

28. Hsu W-C, Liu M-W, Lu T-W. Biomechanical risk factors for tripping during obstacle-crossing with the trailing limb in patients with type II diabetes mellitus. Gait Posture. 2016;45:103-109; doi: 10.1016/j.gaitpost.2016. 01.010 . 